\title{
A Fluorogenic Labeling of Live Viruses
}

Bin Zheng, ${ }^{1, \#}$ Xianhuang Li, ${ }^{1, \#}$ Sheng Xie, ${ }^{2, \#, ~ * ~ S h u o d o n g ~ W a n g, ~}{ }^{2}$ Weiming Lai, ${ }^{2}$ Zhibiao Zhou, ${ }^{2}$ Qing Liu, ${ }^{2}$ Yujiao Yang, ${ }^{2}$ Bei Hou, ${ }^{1}$ Weiwei Ni, ${ }^{3}$ Jinsong Han, ${ }^{3}$ Yong Liu, ${ }^{4}$ Zebing Zeng, ${ }^{2}$ Tao Wang, ${ }^{1,}{ }^{*}$ Ben Zhong Tang $4,5,6,7 *$

${ }^{1}$ Academy of Medical Engineering and Translational Medicine, School of Life Sciences, Tianjin University, Tianjin 300072, China;

${ }^{2}$ State Key Laboratory of Chemo/Biosensing and Chemometrics, College of Chemistry and Chemical Engineering, Hunan University, Changsha 410082, China;

${ }^{3}$ State Key Laboratory of Natural Medicines/National R\&D Center for Chinese Herbal Medicine Processing, College of Engineering, China Pharmaceutical University, Nanjing 210009, Jiangsu, China;

${ }^{4}$ AIE Institute, Guangzhou Development District, Huangpu, Guangzhou 510530, China;

${ }^{5}$ Department of Chemistry, Hong Kong Branch of Chinese National Engineering Research Center for Tissue Restoration and Reconstruction, Institute for Advanced Study and HKUST-Shenzhen Research Institute, SCUTHKUST Joint Research Institute, The Hong Kong University of Science \& Technology, Hong Kong, China;

${ }^{6}$ Center for Aggregation-Induced Emission, State Key Laboratory of Luminescent Materials and Devices, South China University of Technology, Guangzhou 510640, China;

${ }^{7}$ Shenzhen Institute of Molecular Aggregate Science and Engineering, School of Science and Engineering, The Chinese University of Hong Kong (Shenzhen), Shenzhen 518172, China.

\#These authors contributed equally to this work.

*Correspondence: shengxie@hnu.edu.cn (S.X.); wangtaobio@tju.edu.cn (T.W.); tangbenz@cuhk.edu.cn (B.Z.T.)

\begin{abstract}
Viruses are an important class of pathogens that can cause serious infectious diseases. The ability to directly analyze viral particles in real time is essential to understand their pathological functions. In this work, we developed a strategy for fluorogenic labeling of live viruses by using an optimal tetrazolate-functionalized AIEgen (Aggregation-induced emission luminogen), named PBET. Due to its AIE activities, this dye fluoresces weakly when dissolved in aqueous solution, thus providing a dark fluorescence background. The fluorescence turn-on labeling is achieved by binding of PBET molecules into the outmost viral proteins (e.g., structural Capsid protein VP1), which triggers protein-induced fluorescence enhancement (PIFE) of the PBET. Probably due to this unique PIFE/AIE-based sensing strategy, these PBET-labeled viruses can retain infectivity to a large extent, which is a great advantage for biologically friendly labeling. Importantly, this approach can be accomplished by simply mixing the dye and virus samples without any prior modification of the viral particles, and shows high efficiency and stability for both enveloped and non-enveloped viruses. By using PBET-labeled viruses, their early viral infection processes were visualized and monitored over a period of up to 36 hours, including the attachment, entry, and intracellular transport of viruses in living cells. In vivo bioimaging studies on animal models suggest that the fluorescence signal can be used as an indicator of the accumulation of viral particles in organs. This fluorogenic labeling may find uses in imaging studies to combat virus-associated infectious diseases, especially those associated with previously unknown wild-type viruses.
\end{abstract}




\section{Teaser}

The work describes a versatile and bio-compatible strategy for fluorogenic labeling of live viruses, which may find immediate imaging application for combating virus-associated infectious diseases.

\section{TOC}

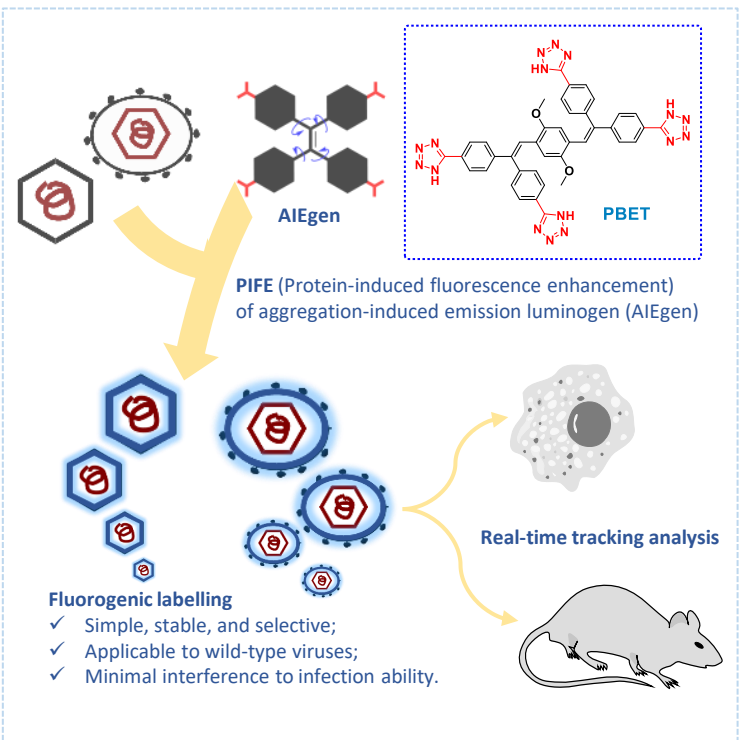

\section{Keywords}

Single-virus tracking, Fluorogenic labeling, Aggregation-induced emission, Protein-induced fluorescence enhancement, Fluorescent probe 


\section{Introduction}

In recent years, infectious diseases caused by novel viruses such as avian influenza, Zika virus disease, and Coronavirus disease 2019 (COVID-19) have posed a great threat to the human society. To prevent and control the spread of viral diseases, it is essential to develop tools to detect and monitor these small-sized viral particles $(20 \sim 300 \mathrm{~nm})(1)$. In this regard, real-time tracking and analysis of viruses can provide a fundamental understanding of their life cycle and help to unravel their pathogenesis (2-7). The process of virus infection into host cells involves multiple stages, different pathways, and many subcellular structures, and is highly complex and therefore challenging to track (8-10). In this context, fluorescence-based imaging has emerged as a powerful spatiotemporal method for visualizing live viruses under physiological conditions (11-13). Fluorescent tags are required to light up these mobile viral particles, and genetic engineering or covalent bioconjugation are currently the main labeling strategies $(1,14)$. For example, the expression of reporter genes for luciferase $(15,16)$, fluorescent proteins (17-20) and fluorophore-attachable peptide tags $(7,21,22)$, has been established to monitor the biological events of viral invasion, replication, and dissemination. However, genetic modification requires specialized techniques, such as recombinant gene technology (23), is time-consuming, generally not well-established for wild-type virus strains, and can also pose a significant safety risk to the operators $(1,14)$. Since viruses are densely packed, the integration of external genes and/or proteins can further affect the genetic stability and infectivity of the virus, resulting in a low level of effective labeling (24). Viruses labeling with organic fluorophores (25-29) and fluorescent nanoparticles $(30,31)$ via covalent bioconjugation is another straightforward approach, but there are still practical problems. Specific conjugation strategies are essential for selective and stable labeling with small fluorophores. In addition, unbound fluorophores must be washed off, otherwise, the spatiotemporal resolution of the freely moving viral particles is compromised. The modification of viruses such as covalent functionalization of proteins and irreversible intercalation to form DNA/RNA-dye complexes, can dampen viral infectivity as well. Moreover, for long-term, realtime tracking analysis of nano-sized viral particles, it is desirable that these fluorescent tags are small-sized, site-specifically labeled, have high brightness and good photo-stability $(1,14)$. 
In contrary to many conventional fluorophores, aggregation-induced emission luminogens (AIEgens) are dark or weakly luminescent in the molecularly solvated or freely dispersed states, but can emit strongly upon aggregation and/or by other effects resulting from restricted intramolecular motions (RIMs) (32). This unique AIE property has been developed as a reliable fluorogenic strategy for chemical sensing, physical visualization and biological imaging (3335). Particularly in bio-imaging of microorganisms (36-38) and living cells, the use of AIEgens can be expected for wash-free fluorogenic labeling, and many of these systems have been approved for long-term fluorescent tracking with high brightness, low background, and good photo-stability $(39,40)$. The exploration of these AIE-based strategies for virus labeling has been relatively lagged off, probably due to the inherent resolution of fluorescent techniques for viral particles and the limited accesses to virus research facilities.

On viewing of the COVID-19 outbreak, we began to screen a library of AIE material for fluorogenic labeling of live viruses, by using non-enveloped Adenovirus serotype 5 (AD5) (41) and Enterovirus D68 (EV-D68) (42), and enveloped Sendai virus (SEV) (43) as model targets (Figure 1a). Surprisingly, we identified two tetrazolate-functionalized AIEgens (TPE-4TA and PBET, Figure 1b) that can elicit a fluorescent turn-on labeling instantly after mixing with viruses. This specific fluorogenic labeling is likely attributed to the protein-induced fluorescence enhancement (PIFE) phenomenon (44-46) of these AIEgens, primarily achieved by specific non-covalent interactions with virus proteins on the outer envelope and capsid. Similar strategy was previously reported for fluorogenic labeling of Serum albumins by using TPE-4TA (47). By using the optimal PBET (which shows greatly improved fluorogenic performance over TPE-4TA) from a library of tetrazolate-functionalized AIEgens in this work, we established a fast, reliable, and selective live virus labeling protocol, and further proof-ofconcept demonstrated this labeling method for real-time tracking analysis of viral infection processes in suspected cellular hosts as well as in animal models. Importantly, this proteinmediated, PIFE-based AIE labeling exhibits little influence on viral infectivity. This simple and efficient strategy is compatible for labeling both enveloped and non-enveloped viruses, thus providing a promising universal method for live virus-related studies, including previously unknown, wild-type viruses. 

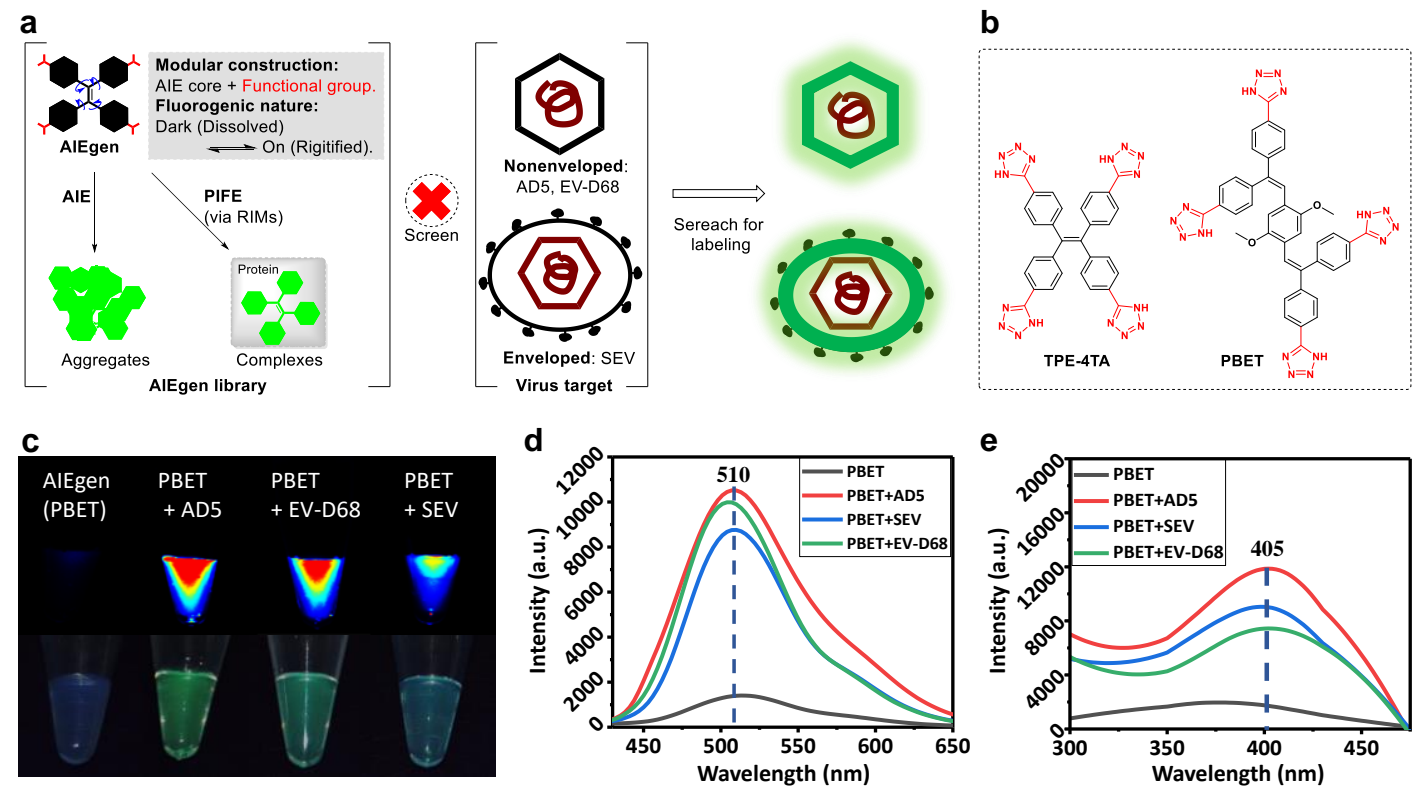

Figure 1. The proposed strategy of using AIEgens to label viruses. (a) Schematic illustration of the principle in terms of screening AIEgens for fluorogenic viral labeling. PIFE: Protein-induced Fluorescence Enhancement. RIMs: Restriction of Molecular Motions. (b) Chemical structures of the TPE-4TA and PBET dyes. (c) Fluorescent images of the PBET solution and the mixtures with viruses of different strains. (d) Corresponding fluorescence excitation spectrum and (e) emission spectrum of the samples in (c). [PBET] $=5 \mu \mathrm{M}$; [Virus $]=10^{7} \mathrm{TCID}_{50} \mathrm{~mL}^{-1}$.

\section{Results and discussion}

\section{Screening of AIEgens for viral labeling}

The initial screening involved a library of diverse AIEgens developed in our laboratory, especially those with good aqueous solubility. These AIEgens fluoresce only weakly or even darkly in aqueous solution, thus providing a low fluorescence background. Both nonenveloped (AD5 and EV-D68) and enveloped (SEV) viruses were used as the model target. The screening experiments were performed in culture medium, DMEM buffer, and deionized water solutions, respectively. By simply following the fluorescence change before and after mixing with viruses (Supplementary Figure 1), we first discovered that tetrazolate-functonalized tetraphenyletheylene TPE-4TA (Figure 1b) could slightly light up these virus solutions. But these labelled viruses were not bright enough under fluorescence microscopy. Subsequently, we expanded a library of tetrazolate-functionalized AIEgens (Supplementary Figure 2) through combinatorial organic synthesis, i.e., by introducing tetrazolate groups into different types of AIE-active cores in a modular way (48), and finally found out PBET as an optimal dye 
(Supplementary Figure 3 for synthesis; Supplementary Figures 4-5 for compound characterization) which exhibited much enhanced fluorogenic response (Fluorescence turn-on ratio at $510 \mathrm{~nm}>10$ times, excitation: $405 \mathrm{~nm}$ ) towards viruses (Figures 1c-e).

\section{Characterization of the fluorogenic labeling}

We next characterized the fluorogenic labeling of these viruses using PBET. When an aliquot of wild-type natural viruses was added into a $5 \mu \mathrm{M}$ PBET solution, a strong green fluorescence could be detected from the mixture under $365 \mathrm{~nm}$ excitation. The fluorescence turn-on effect was clearly shown under the imaging system (Figure 1d), and the response reached a steady state instantaneously after mixing ( $<1 \mathrm{~min})$. The green fluorescence emission of the mixture was peaked at around $510 \mathrm{~nm}$ for the three different virus strains (Figure 1e). Comparably, the dye solution without viruses emitted at $510 \mathrm{~nm}$ but faintly. The enhancement of fluorescence intensity at $510 \mathrm{~nm}$ exceeds 8 times. Their maximum long-wavelengths for excitation were peaked at about $405 \mathrm{~nm}$, which was red-shifted from $375 \mathrm{~nm}$ of the pure PBET solution (Figure 1f). The fluorescence titration studies revealed that when either the titers of viruses or the concentration of PBET was increased, the corresponding fluorescence intensity increased in a quasi-linear way (Figure 2, and also see Supplementary Figure 6: AD5; Supplementary Figure 7: EV-D68; Supplementary Figure 8: SEV). For example, the increased titers of AD5 viruses enhanced the fluorescence signal of mixture with $5 \mu \mathrm{M}$ PBET (Figures $2 \mathrm{a}-\mathrm{b})$; the peak intensity at $510 \mathrm{~nm}$ showed a good linearity $\left(\mathrm{R}^{2}=0.995\right)$ towards the titers of viruses in the range of $10^{5}-10^{7} \mathrm{TCID}_{50} \mathrm{~mL}^{-1}$ (Figure 2c), indicating that this labeling strategy can be used for quantitative analysis of viruses. A similar fluorogenic labeling was also achieved for non-enveloped EV-D68 viruses (Figure 2e-g), and enveloped SEV viruses (Figures 2i-k). We then characterized the viral particles in solutions before/after the labeling using dynamic light scattering. The size distributions of these three AIE-labeled virus samples displayed a pattern comparable to that of the corresponding non-labeled virus samples with a slight increase in averaged peak sizes (Figure 2d for AD5; Figure 2h for EV-D68; Figure 21 for SEV), indicating a freely dispersed state of these AIE-labeled viruses in solutions. These also correlated well with the TEM (Transmission electron microscopy) characterization which showed viral particles with characteristic morphology after labeling (Figures 2m-n); aggregates 
of AIEgens were moreover not observed. Under the typical labeling condition, the surface zeta potentials $(\xi)$ of viral particles all slightly decreased after treating with the dye but retained to be negatively charged (Figure 20): from $-755 \mathrm{mV}$ to $-255 \mathrm{mV}$ for $\mathrm{AD}$, from $-484 \mathrm{mV}$ to -287 $\mathrm{mV}$ for EV-D68, and from $-776 \mathrm{mV}$ to $-472 \mathrm{mV}$ from SEV. These results show that PBET can directly illuminate viruses without prior chemical modification of viral cells, or cumbersome labeling strategies such as the use of antibodies, providing an attractive strategy for later tracking studies of viral infections.
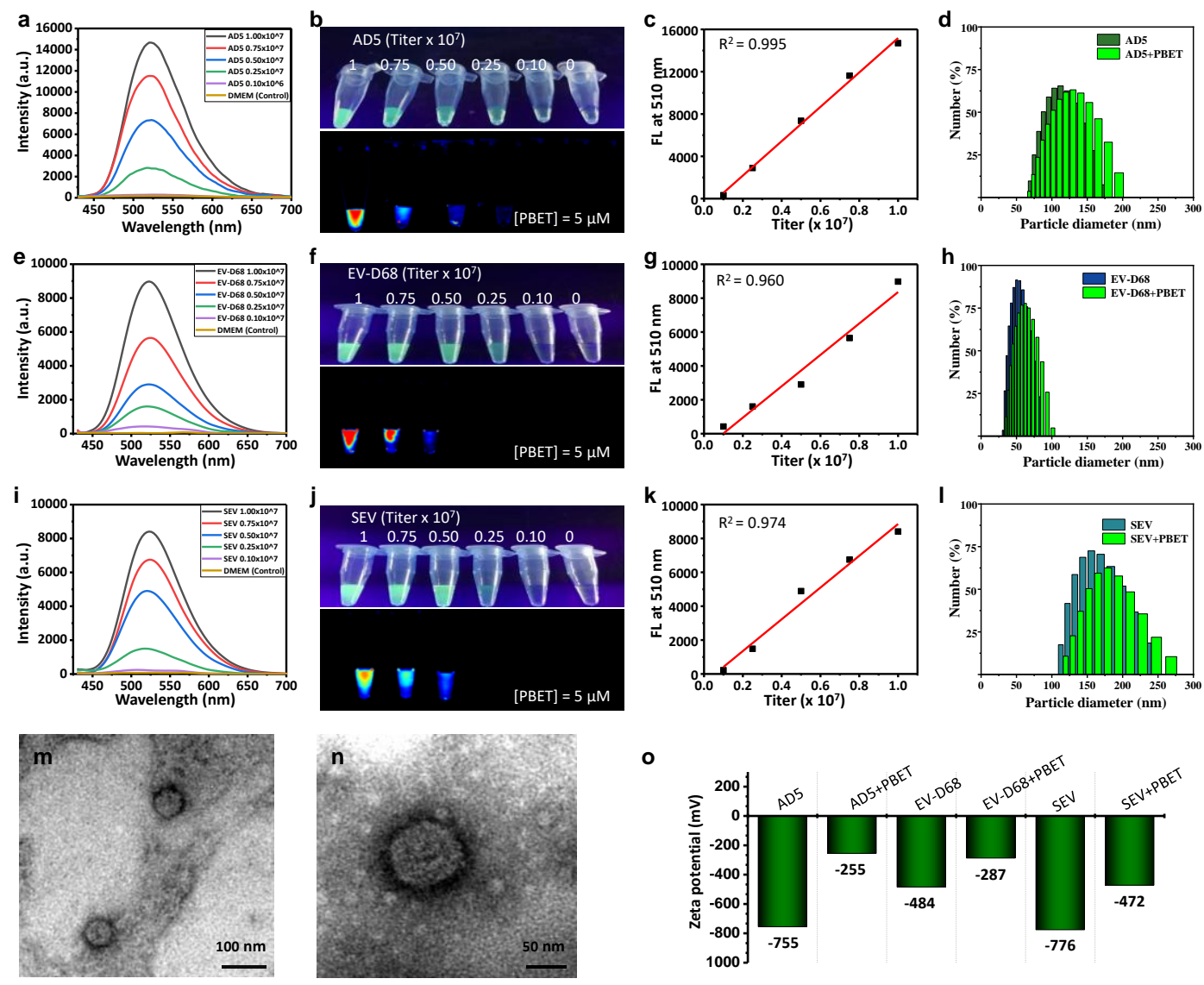

Figure 2. Characterization of the fluorogenic labeling for nonenveloped and enveloped viruses. (a-b) Fluorescent titration of non-enveloped AD5 viruses into a $5 \mu \mathrm{M}$ PBET solution and (c) corresponding fluorescence intensity against titers of the viral particles. (e-f) Fluorescent titration of non-enveloped EVD68 viruses into a $5 \mu \mathrm{M}$ PBET solution and (g) corresponding fluorescence intensity against titers of the viral particles. (i-j) Fluorescent titration of enveloped SEV viruses into a $5 \mu \mathrm{M}$ PBET solution and (k) corresponding fluorescence intensity against titers of the viral particles. $(\mathrm{d} / \mathrm{h} / \mathrm{l})$ Size changes before/after labeling with PBET, measured by DLS. (m/n) TEM characterization of EV-D68 (m) and AD 5 (n) after labeling with BPET. (o) Surface zeta potentials $(\xi)$ of viral particles before/after labeling with PBET, measured by DLS. [BPET] $=2.5 \mu \mathrm{M}$. FL: fluorescence. 


\section{PBET binds to proteins of viral particles and turn on the fluorescence}

To reveal which components of the virus interacts with PBET to induce the fluorescence emission, we used purified components from the virus lysate to mix with the dye independently, including the Capsid protein VP1 from EV-D68, the RNA component obtained by reverse transcription of the EV-D68 plasmid, and the DNA component from the AD5 viral backbone plasmid. As shown in Figure 3a, while there was no fluorescence change for the corresponding RNA and DNA components, a similar fluorogenic AIE phenomenon was observed when using purified Capsid protein VP1 in solutions. Fluorescence titration studies clearly showed that the fluorescence became stronger with increasing VP1 concentration (Figure 3d-e), showing a good linear relationship with $\mathrm{R}^{2}=0.944$ (Figure 3f). These fluorescence-based properties of PBETVP1 complexes are essentially similar to those of PBET complexes with viral particles (Figure 2), which is direct evidence that PBET may also bind specifically to the outermost proteins of the virus and then light up the viral particles. This kind of protein-induced fluorescence enhancement (PIFE) phenomenon was also observed for TPE-4TA, which binds specifically to albumin proteins through tetrazole-mediated hydrogen-binding interactions with the basic amino groups in the protein pockets (47). We then conducted a molecular docking study to predict and elucidate the interaction between the PBET dye and the VP1 protein (PDB code: 4WM7, from Human Enterovirus D6) (49). As shown in Figure 3b/c, the small PBET molecule entered protein (Figure 3b), and three out of four tetrazole groups were observed to form ionic and hydrogen-bonding molecular interactions with Thr97, Ala169, and Met187 in the active site region of VP1 protein (Figure 3c). Multiple tetrazole groups help restrict the free motions of the AIE-cored fluorogen, which suppresses the excited-state energy-dissipating pathway to elicit a fluorescence turn-on sensing. Since among the $>10$ tetrazolate-functionalized AIEgens (Supplementary Figure 2), only PBET showed a good fluorescence turn-on response to viruses in this case, we suspect that PBET may enter and binds to the pockets of certain viral proteins in a specific manner. Besides the Capsid protein VP1 from EV-D68, we do not have other purified viral proteins currently, especially those from SEV or AD5, to further evaluate the generality of the fluorogenic labeling for both enveloped and non-enveloped viruses. 

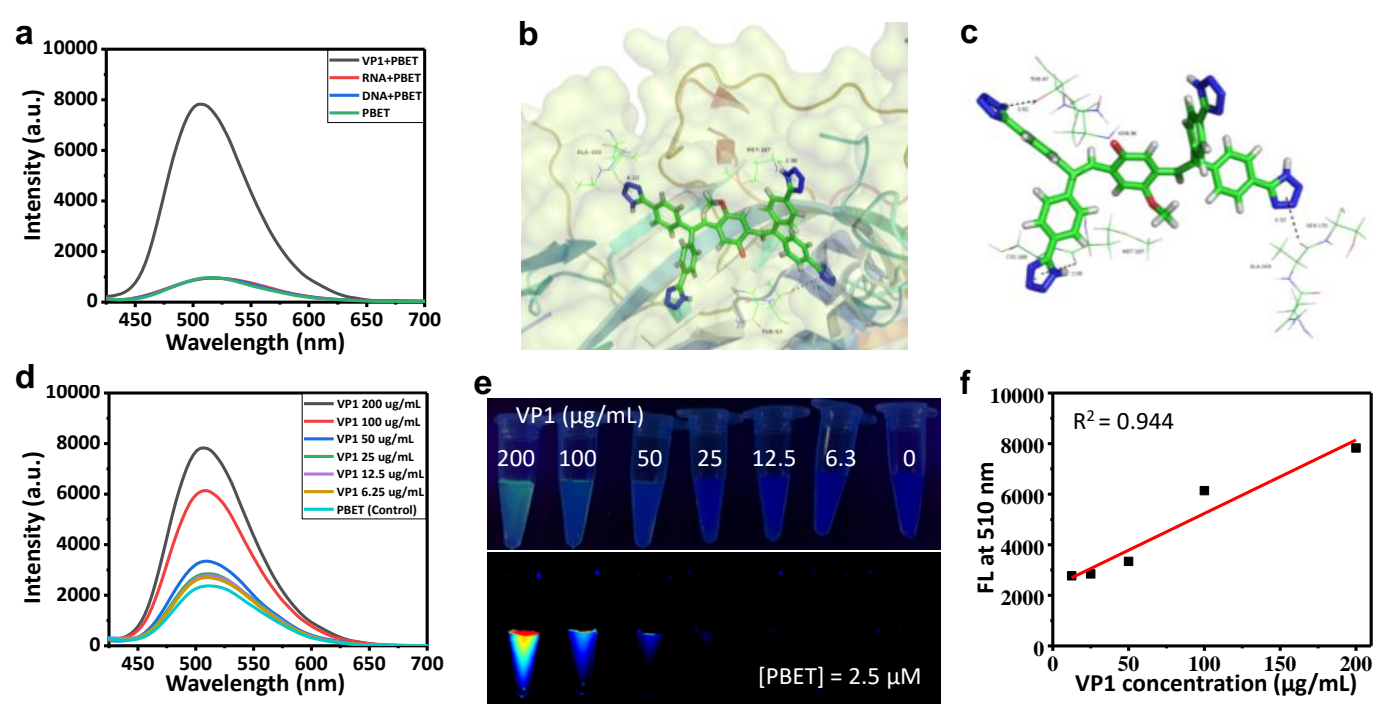

Figure 3. Investigation of the fluorogenic labeling mechanism. (a) Fluorescence spectra of PBET mixed with purified viral components, including Capsid protein VP1 (structural protein from EVD68), DNA and RNA. (b-c) Molecular docking analysis showing the Putative binding mode of PBET, based on the crystal structure of VP1 protein (PDB code: 4WM7). These figures were generated using PyMol software (http://www.pymol.org/). (d-f) Fluorescence titration study of PBET (2.5 $\mu \mathrm{M})$ with different VP1 concentrations: (d) fluorescence spectrum, (e) corresponding fluorescence image, and (f) the fluorescence peak intensity at $510 \mathrm{~nm}$ against the concentration of VP1.

\section{Influence of PBET labeling on viral infectivity}

Does this labeling have a negative influence on viral infectivity? To evaluate this possibility, titers were calculated to compare batches of labeled and wild-type viruses regarding viral infectivity. Wild-type viruses with a titer of $10^{7} \mathrm{TCID}_{50} \mathrm{~mL}^{-1}$ were selected and tested for viral titers after labeling with PBET. Both were able to detect the virus at $10^{7}$. This indicates that the use of PBET labeling has little effect on the infectivity of the virus.

\section{Real-time tracking of virus infection into cells}

Next, we used these fluorescently labelled viruses as a model to visualize the process of viral infection into susceptible cells. Cells were first incubated with labeled viruses for $30 \mathrm{~min}$. Afterwards, they were washed with fresh DMEM and then observed under a fluorescence microscope. In these cases, a number of emissive viral particles (arrow highlighted) can be identified, shown as close to the membrane of 293A cells (Red, stained by Dil) (Figure 4a). In other tests, fluorescent EV-D68 and SEV viral particles could also be observed similarly 
(Supplementary Figures 9-10). These results imply that the labeling strategy will not affect the virus binding to host cells. By contrast, when PBET was directly added to the cell culture medium in a control experiment, no blue-emissive particles could be identified.
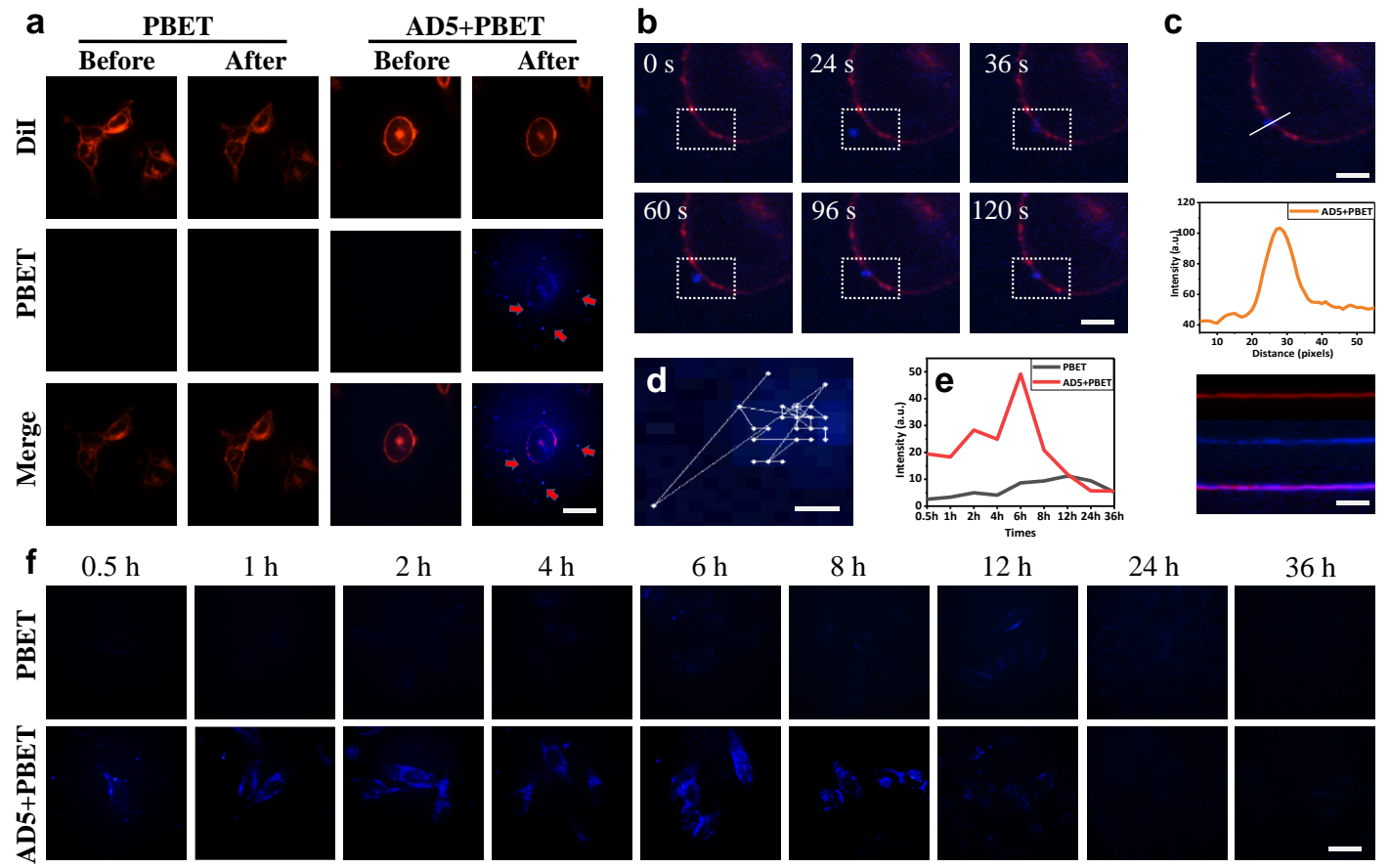

Figure 4. Fluorescent tracking analysis of PBET-labeled AD5 viral particles into living 293A cells.

(a) Confocal fluorescence images of cell after incubation with labeled AD5 viruses. Red: Dil-labeled cell membrane, blue: PBET-labeled AD5 viral particles. Scalebar: $25 \mu \mathrm{m}$. (b) Video snapshots of the entry of a PBET-labeled virus via confocal fluorescence images, Scalebar: $5 \mu \mathrm{m}$. (c) Kymograph of the binding site of the virus, including fluorescence intensity curve (Middle) of the site of virus. Scalebar: $1 \mu \mathrm{m}$. The distance between two neighboring pixels is about $12.5 \mathrm{~nm}$. (d) Trajectory of viral diffusion from sequential images in the b) section. (e) Time-dependent fluorescence intensity change of BPET-labeled AD5 virus in the (f) section. (f) Long-term fluorescent tracking of PBETlabeled AD5 viruses with living 293A cells; the control group used the PBET dye solution without viruses. Scalebar: $25 \mu \mathrm{m}$.

Real-time tracking analysis was carried out to visualize the attachment, entry, and intracellular transport of BPET-labeled viruses in living cells (Figure 4 for AD5; Supplementary Figure 9 for EV-D68; Supplementary Figure 10 for SEV). The infection process of individual virus was monitored at 12-s intervals (Figure 4b). We could catch up a representative snapshot to show exactly that a viral particle was sitting on the cell membrane (Figure 4c). The middle image in Figure $4 \mathrm{c}$ showed the fluorescence intensity curve of the site of virus, which further confirmed 
that the fluorescent dot was a virus. The trajectory of this single virus for the whole process was extracted from the video, showing the movements of a virus around/on the cell membrane before the internalization (Figure 4d).

PBET-labeled viruses were followed up to $36 \mathrm{~h}$ after incubation to monitor the entire early post-infection process. After incubating with $293 \mathrm{~A}$ cells at $37^{\circ} \mathrm{C}$ for $0.5 \mathrm{~h}$, fluorescently labeled AD5 viruses were observed on the cell membrane, and accumulated more and more over time to reach maximum in $\sim 2 \mathrm{~h}$, as reflected by the corresponding fluorescence intensity (Figure $4 \mathrm{f}$ ). Thereafter, the fluorescence from viruses on the cell membrane became weaker and weaker, lasting for about 8-12 h. However, starting from the first hour, these PBET-labeled viral particles were detected in the cytoplasm, indicating that they had been internalized into the cells to form endosomes. After that, the intracellular fluorescence began to decrease (Figure 4e). The loss of virus-associated fluorescence is mainly attributed to the disassembly of these PBETlabeled viruses. During this phase, viruses can remove their envelope and/or capsid in the endosome, release their DNA or RNA, and enter the hidden infection phase. Along with the uncoating of capsid or/and envelop, the PBET dye detached from the virus protein (Especially when the protein is digested and/or degraded by the cell) and be solubilized, resulting in decreased fluorescence. We observed a similar phenomenon with PBET-labeled EV-D68 and SEV viruses when infecting RD cells (Supplementary Figures 9-10). As above revealed, these viruses bind to the receptors on the cell membrane within $1 \mathrm{~h}$ after incubating with the cells, and are subsequently endocytosed in another $1 \mathrm{~h}$ and form endosomes in the cytoplasm. After 8-12 h of infection, the viral envelope is largely un-coated, and the virus begins to enter the hidden period of replication. The infection process of these labeled viruses is broadly similar to those in references (1-7).

\section{In vivo bioimaging of viruses in experimental animals}

Finally, we evaluated these PBET-labeled viruses in the in vivo bioimaging study using mice models. All these PBET-labeled viruses gave significantly intensive signals under the in vivo luminescence imaging system compared with the control groups (Figure 5a). In vivo imaging showed intense fluorescence at the subcutaneous injection sites of mice for the three groups using PBET-labeled viruses, but not the group using a pure PBET solution (Figure 5b). 
In another group of animal experiments, these labelled virus samples were firstly injected into the BALB/C nude mice by caudal vein. At $12 \mathrm{~h}$ after tail vein injection, the major internal organs of the mice were taken out after euthanasia treatment, and studied under the in vivo imaging instrument. Fluorescence signals were detected in all liver organs. Compared with the control PBET group, the liver organs from the three kinds of different PBET+virus groups exhibit strong luminescence signals, with the PBET+EV-D68 group being the most intense (Figure 5c). The group using PBET+EV-D68 exhibited a relatively more dispersed distribution of fluorescence. In the mice injected with PBET+EV-D68 virus, notable fluorescence signals were also detected in the heart and kidney organs. Subsequently, we performed quantitative real-time PCR (q-PCR) measurements on these organ samples to quantify virus accumulation and confirmed that the virus accumulation pattern (Figure 5d) correlated well with that from the bioimaging result (Figure 5c), which further validated the reliability of this fluorogenic labeling method. Based on this observation principle, we can conclude that the three different virus strains show quite different infection patterns in terms of virus accumulation. For example, AD5 viruses didn't infect organs other than the liver, while SEV viruses could enter the lungs in large numbers within 12 hours.

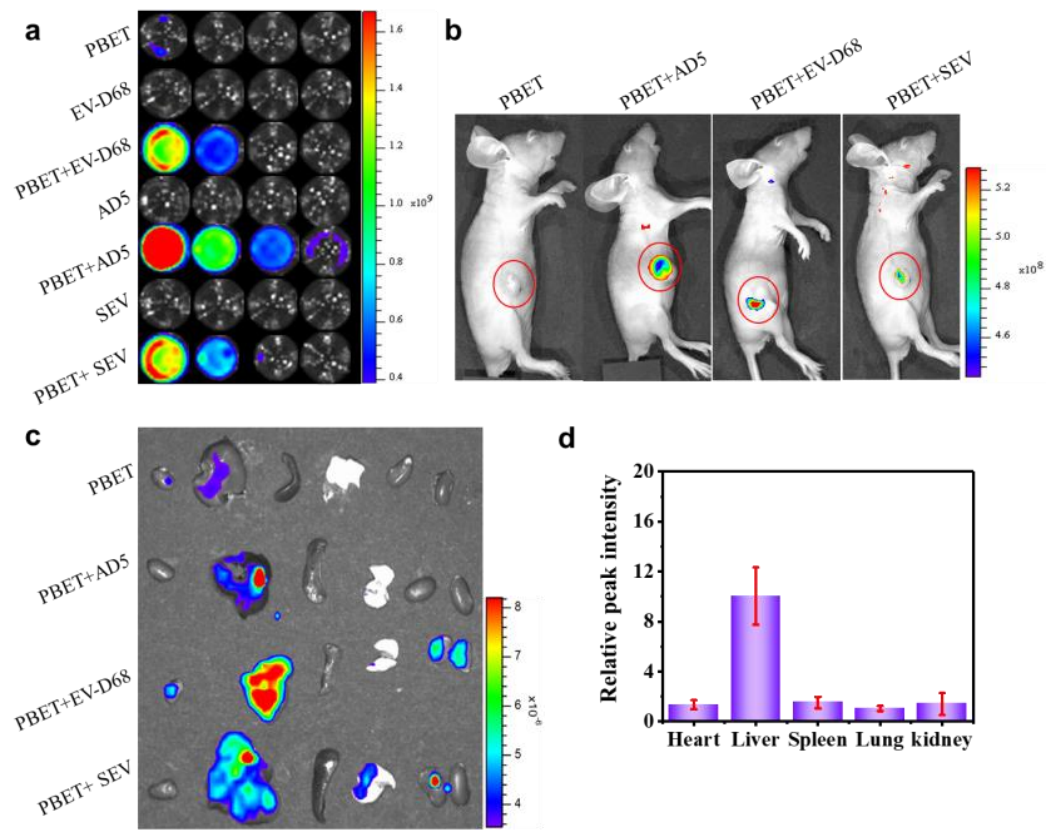

Figure 5. Fluorescence bioimaging of virus infection in the animal model. (a) Fluorescent images of samples by the in vivo imaging system. (b) Fluorescence bioimaging of mice after subcutaneous 
injection of different PBET+virus samples. (c) Fluorescence images of excised organs obtained at $12 \mathrm{~h}$ after tail vein injection of different PBET+virus samples. (d) Relative intensity of viral content in excised organs obtained at $12 \mathrm{~h}$ after tail vein injection of PBET-labeled EV-D68, measured by q-PCR. ${ }^{*} \mathrm{P}<0.05 ; * * \mathrm{P}<0.01$.

\section{Conclusion}

In this work, we report an AIE-based fluorogenic labeling strategy for single virus analysis that that is simple to operate, efficient, reproducible, minimally interferes with viral infectivity, does not require any prior modification of viral particles, and can be widely adapted to enveloped and non-enveloped virus strains. The fluorescence turn-on labeling can be accomplished by simple mixing of PBET dye solutions with viral samples, and moreover, enables washing-free imaging in subsequent experiments. Experimental and computational investigations suggest that this unique labeling is likely to be achieved by specific binding of PBET molecules to the outmost proteins on the viral Capsid or envelop, resulting in proteininduced fluorescence enhancement (PIFE) of multiple tetrazolate-functionalized AIE-active PBET via the RIMs mechanism. Particle characterization showed that PBET-labeled viruses could largely retain their morphology, surface charge, and physical aggregate state. We performed proof-of-principle studies using PBET-labeled viruses to visualize the viral infection process in real-time into susceptible living cells over a period of $>36$ hours. In addition, PBETlabeled viruses are compatible with live animal models for in vivo bioimaging studies, showing the potential to visualize the accumulation process of viruses in different organs. As demonstrated, this strategy not only confers a highly valuable traceability on viruses, but also maximally preserves the fluorogenic properties of the AIE sensor and the infectivity of viruses, which is essential for advanced imaging analysis of viral particles at different cellular, organ, and animal levels. Importantly, and interestingly, this strategy appears to be a general yet specific molecular tool for labeling both enveloped and non-enveloped viruses, especially wildtype strains. Thus, this tool may find immediate application in studies to combat virusassociated infectious diseases. 


\section{Materials and Methods}

Virus. Adenovirus serotype 5 (AD5) strain is a kind gift from Prof. Zhuozhuang Lu's group at Chinese Center for Disease Control and Prevention. The Sendai virus (SEV) BB1 strain a kind gift from Dr. Lishu Zheng's group at Chinese Center for Disease Control and Prevention. The Enterovirus D68 strain (EV-D68; GenBank accession number KU844179.1) is a kind gift from Prof. Xiaofang Yu's group at Johns Hopkins University.

AIEgen. These AIEgens used are either developed in our lab, or kindly donated from AIEgen Co.. Many tetrazole-functionalized AIEgens are newly designed and prepared by organic synthesis. Please see Supplementary Figure 3 for the preparation of the PBET AIEgen.

Cells and animals. Human Embryonic Kidney 293A (293A) cells and Rhabdomyosarcoma (RD) cells were cultured in high-glucose Dulbecco modified Eagle's medium (DMEM; Invitrogen), supplemented with $10 \%$ fetal bovine serum (FBS; Ausbian), $1 \%$ penicillin/streptomycin (Invitrogen) at $37{ }^{\circ} \mathrm{C}$ and $5 \% \mathrm{CO}_{2}$. Healthy female BALB/C nude mice were purchased from HFK Technology Co., Ltd. (Beijing).

Characterization. The $\zeta$-Potential and size distributions of viruses were determined by a zetasizer nanoseries (Malvern instrument). The shape and morphology of virus samples were determined via transmission electron microscopy (TEM, Hitachi HT7700). The fluorescence spectrum of virus samples was investigated on an EnSpire Multilabel Reader. In vitro fluorescence imaging was conducted by putting these PBET-labeled viruses into glass bottom culture Dishes. The luminescent signal was recorded by IVIS Spectrum In Vivo Imaging System (IVIS®Spectrum BL, PerkinElmer).

Preparation of virus for labeling. EV-D68 was prepared in RD cell culture, as previously reported (50). AD5 was prepared in 293A cell culture by incubation with the viral suspension, as previously reported (51). Once cells displayed $80 \%$ cytopathic effect, cell culture medium were exposed to three freeze-thaw cycles, followed by centrifuging at $2000 \times \mathrm{g}$ for $15 \mathrm{~min}$ at $4{ }^{\circ} \mathrm{C}$ to remove cellular debris. The culture supernatants containing viruses were carefully 
harvested by ultracentrifugation through SDGC at 110, $000 \mathrm{~g}$ for $2 \mathrm{~h}$ in an SW41 rotor (Beckman Coulter Inc., Germany). After removing sucrose, viruses were sub-packaged and stored at $-80^{\circ} \mathrm{C}$. SEV viruses were propagated in 10-day-old embryonated eggs for $48 \mathrm{~h}$ at $37^{\circ} \mathrm{C}$, as previously reported (52).Subsequently, viruses in allantoic fluid were harvested and purified by SDGC at $110000 \mathrm{~g}$ in an SW 41 rotor for $2 \mathrm{~h}$ at $4^{\circ} \mathrm{C}$.

Virus labeling. The PBET powders were firstly dissolved in fresh medium to have a concentration of $10^{-5} \mathrm{M}$. The purified viruses were dispersed in another fresh medium or DMEM to have certain concentrations. In a typical labeling, the PBET solution was then added into the virus solution in equal volume $(20 \mu \mathrm{L})$.

Molecular docking. Molecular docking of PBET into the crystal structure of Human Enterovirus D68 in complex with Pleconaril was carried out using SYBYL-X version 2.1.1 software suite (Tripos, Inc., St. Louis, MO). The X-ray structure of Human Enterovirus D68 was downloaded from the Protein Data Bank (PDB code: 4WM7) and was modified by adding hydrogen atoms and removing water as well as co-crystallized substrate (Pleconaril). The active site was defined as all the amino acid residues confined within a $5 \AA$ radius sphere centered about Pleconaril, and the composite structure without original ligand was utilized as the insilico model for docking studies. Default parameters and values within the minimization dialogue were used except where otherwise mentioned. The docked conformations of ligands were evaluated and ranked using Surflex-Dock and four scoring functions implemented in the CSCORE software module within the SYBYL-X environment. The CSCORE module allowed consensus scoring that integrated multiple well-known scoring functions such as ChemScore, D-Score, G-Score and PMF-Score to evaluate docked ligand conformations.

Evaluation of viral infectivity. The infectivity of TPE-labeled viruses was quantified by $50 \%$ tissue culture infective dose $\left(\mathrm{TCID}_{50}\right)$. In the $\mathrm{TCID}_{50}$ assay, cells (RD, 293A and MDCK) were cultured in 96-well plates in culture medium until the cells reached $80-90 \%$ confluence. Labeled and non-labeled viruses were $10^{1}$-fold diluted ranging from $10^{-4}$ to $10^{-9}$ in infection culture media. 
Fluorescence in vivo bioimaging. Animal experiments were performed in accordance with the statutory requirements of People's Republic of China (GB14925-2010). A solution (100 $\mu \mathrm{L})$ of correspondingly labeled viruses were injected into each mouse by subcutaneous injection and tail vein.

\section{q-PCR detection of viral content.}

Total RNA was extracted using Trizol (Qiagen). RNAs were reverse transcribed to cDNA using the TransScript First-Strand cDNA Synthesis SuperMix Kit in accordance with the manufacturer's instructions. Quantitative real-time PCR (q-PCR) was performed using TransStart Top Green q-PCR SuperMix (TransGen, China) in a $20 \mu \mathrm{L}$ reaction volume $(10 \mu \mathrm{L}$ TransStart Top Green q-PCR SuperMix, $200 \mathrm{mM}$ forward and reverse primers, $2 \mu \mathrm{L}$ cDNA template). The following cycling conditions: $94^{\circ} \mathrm{C}$ for $30 \mathrm{~s}$ and 40 cycles of $94^{\circ} \mathrm{C}$ for $5 \mathrm{~s}$ and $60^{\circ} \mathrm{C}$ for $30 \mathrm{~s}$. The reaction was run on a LightCycler 96 Real-Time PCR machine (Roche), and levels of gene mRNAs were normalized to GAPDH mRNA.

Image analysis. Imaging data from virus solutions, cultured cells, and animals were processed using ImageJ 1.52p software (NIH). The trajectories of virus and time courses of fluorescence intensity were reconstructed from raw images by aligning the coordinates and intensity of spots representing virus and corresponding cellular endocytic structures in each frame with ImagingPro-Plus software (Media Cybernetics). Only the integral trajectories within the focal plane were processed in quantitative single-virus tracking analysis. The statistical analyses were performed using GraphPad Prism 7 and OriginPro 9.1. Two-tailed Student's t-test, two-way analysis of variance (ANOVA) with Bonferroni's multiple comparisons test and one-way ANOVA with Tukey's multiple comparisons test were performed. ${ }^{*} \mathrm{P}<0.05$ and ${ }^{* *} \mathrm{P}<0.01$ (Figure 5). The graphs were generated using OriginPro 9.1 (OriginLab).

\section{Conflict of interest}

The authors declare no conflict of interest. 


\section{References}

1. Liu SL, et al. (2020) Single-Virus Tracking: From Imaging Methodologies to Virological Applications. Chem Rev 120(3):1936-1979.

2. Brandenburg B \& Zhuang X (2007) Virus trafficking - learning from single-virus tracking. Nat Rev Microbiol 5(3):197-208.

3. Jouvenet N, Bieniasz PD, \& Simon SM (2008) Imaging the biogenesis of individual HIV-1 virions in live cells. Nature 454(7201):236-240.

4. Grove J (2014) Super-Resolution Microscopy: A Virus' Eye View of the Cell. Viruses 6:13651378.

5. Qin C, et al. (2019) Real-time dissection of dynamic uncoating of individual influenza viruses. Proc Natl Acad Sci U S A 116(7):2577-2582.

6. Inamdar K, Floderer C, Favard C, \& Muriaux D (2019) Monitoring HIV-1 Assembly in Living Cells: Insights from Dynamic and Single Molecule Microscopy. Viruses 11(1).

7. Boersma S, et al. (2020) Translation and Replication Dynamics of Single RNA Viruses. Cell 183(7):1930-1945 e1923.

8. Conner SD \& Schmid SL (2003) Regulated portals of entry into the cell. Nature 422(6927):3744.

9. Mercer J, Schelhaas M, \& Helenius A (2010) Virus entry by endocytosis. Annu Rev Biochem 79:803-833.

10. Sun E, He J, \& Zhuang XW (2013) Live cell imaging of viral entry. Current Opinion in Virology 3(1):34-43.

11. Kukura P, et al. (2009) High-speed nanoscopic tracking of the position and orientation of a single virus. Nat Methods 6(12):923-927.

12. Sakin V, Paci G, Lemke EA, \& Muller B (2016) Labeling of virus components for advanced, quantitative imaging analyses. FEBS Lett 590(13):1896-1914.

13. Parveen N, Borrenberghs D, Rocha S, \& Hendrix J (2018) Single Viruses on the Fluorescence Microscope: Imaging Molecular Mobility, Interactions and Structure Sheds New Light on Viral Replication. Viruses 10(5). doi:10.3390/v10050250.

14. Huang LL \& Xie HY (2014) Progress on the labeling and single-particle tracking technologies of viruses. Analyst 139(13):3336-3346.

15. Pan W, et al. (2013) Visualizing influenza virus infection in living mice. Nat Commun 4:2369.

16. Karlsson EA, et al. (2015) Visualizing real-time influenza virus infection, transmission and protection in ferrets. Nat Commun 6:6378.

17. Baulcombe DCC, S.; Santa Cruz, S. (1995) Jellyfish Green Fluorescent Protein as a Reporter for Virus Infections. Plant J. 7.

18. Arhel N, et al. (2006) Quantitative four-dimensional tracking of cytoplasmic and nuclear HIV1 complexes. Nat Methods 3(10):817-824.

19. Hubner W, et al. (2009) Quantitative 3D video microscopy of HIV transfer across $\mathrm{T}$ cell virological synapses. Science 323(5922):1743-1747.

20. Miyauchi K, Kim Y, Latinovic O, Morozov V, \& Melikyan GB (2009) HIV enters cells via endocytosis and dynamin-dependent fusion with endosomes. Cell 137(3):433-444.

21. Liu AA, et al. (2016) Simultaneous Visualization of Parental and Progeny Viruses by a CapsidSpecific HaloTag Labeling Strategy. ACS Nano 10(1):1147-1155.

22. Zheng LL, Li CM, Zhen SJ, Li YF, \& Huang CZ (2016) His-tag based in situ labelling of 
progeny viruses for real-time single virus tracking in living cells. Nanoscale 8(44):18635-18639.

23. Shaner NC, Steinbach PA, \& Tsien RY (2005) A guide to choosing fluorescent proteins. Nat Methods 2(12):905-909.

24. Muller B, et al. (2004) Construction and characterization of a fluorescently labeled infectious human immunodeficiency virus type 1 derivative. J Virol 78(19):10803-10813.

25. Seisenberger G, et al. (2001) Real-time single-molecule imaging of the infection pathway of an adeno-associated virus. Science 294(5548):1929-1932.

26. Zhou P, et al. (2012) Multicolor labeling of living-virus particles in live cells. Angew Chem Int Ed Engl 51(3):670-674.

27. Wen LL, Y.; Zhang, Z. L.; Lu, W.; Lv, C.; Chen, Z. L.;Wang, H. Z.; Pang, D. W. (2016) Intracellular Self-Assembly Based Multi-Labeling of Key Viral Components: Envelope, Capsid and Nucleic Acids. Biomaterials 99:24-33.

28. Li Q, et al. (2017) Single-Particle Tracking of Human Immunodeficiency Virus Type 1 Productive Entry into Human Primary Macrophages. Acs Nano 11(4):3890-3903.

29. Wang IH, Burckhardt CJ, Yakimovich A, \& Greber UF (2018) Imaging, Tracking and Computational Analyses of Virus Entry and Egress with the Cytoskeleton. Viruses 10(4), doi:10.3390/v10040166.

30. Marjomaki V, et al. (2014) Site-specific targeting of enterovirus capsid by functionalized monodisperse gold nanoclusters. Proc Natl Acad Sci U S A 111(4):1277-1281.

31. Liu SL, Wang ZG, Zhang ZL, \& Pang DW (2016) Tracking single viruses infecting their host cells using quantum dots. Chem Soc Rev 45(5):1211-1224.

32. Mei J, Leung NL, Kwok RT, Lam JW, \& Tang BZ (2015) Aggregation-Induced Emission: Together We Shine, United We Soar! Chem Rev 115(21):11718-11940.

33. Xie S, Wong AYH, Chen S, \& Tang BZ (2019) Fluorogenic Detection and Characterization of Proteins by Aggregation-Induced Emission Methods. Chem. Eur. J. 25(23):5824-5847.

34. Niu GL, et al. (2020) AIE luminogens as fluorescent bioprobes. Trac-Trend Anal Chem 123:115769.

35. Feng GX, Kwok RTK, Tang BZ, \& Liu B (2017) Functionality and versatility of aggregationinduced emission luminogens. Appl Phys Rev 4(2), doi:10.1063/1.4984020.

36. Xie S, et al. (2017) Design and synthesis of theranostic antibiotic nanodrugs that display enhanced antibacterial activity and luminescence. P Natl Acad Sci USA 114(32):8464-8469.

37. Xiong LH, et al. (2018) Ultrasensitive Virion Immunoassay Platform with Dual-Modality Based on a Multifunctional Aggregation-Induced Emission Luminogen. ACS Nano 12(9):9549-9557.

38. Jing J, et al. (2020) Co-assembly of HPV capsid proteins and aggregation-induced emission fluorogens for improved cell imaging. Nanoscale 12(9):5501-5506.

39. Liang J, Tang BZ, \& Liu B (2015) Specific light-up bioprobes based on AIEgen conjugates. Chem Soc Rev 44(10):2798-2811.

40. Qian J \& Tang BZ (2017) AIE Luminogens for Bioimaging and Theranostics: from Organelles to Animals. Chem-Us 3(1):56-91.

41. Kalyuzhniy O, et al. (2008) Adenovirus serotype 5 hexon is critical for virus infection of hepatocytes in vivo. Proc Natl Acad Sci US A 105(14):5483-5488.

42. Sun J, Hu XY, \& Yu XF (2019) Current Understanding of Human Enterovirus D68. Viruses 11(6):490.

43. Heath RB (1979) The pathogenesis of respiratory viral infection. Postgrad Med J 55(640):122- 
127.

44. Hwang H \& Myong S (2014) Protein induced fluorescence enhancement (PIFE) for probing protein-nucleic acid interactions. Chem Soc Rev 43(4):1221-1229.

45. Kim H, et al. (2018) Protein-induced fluorescence enhancement for a simple and universal detection of protein/small molecule interactions. Rsc Advances 8(70):39913-39917.

46. Rashid F, et al. (2019) Initial state of DNA-Dye complex sets the stage for protein induced fluorescence modulation. Nat Commun 10(1):2104.

47. Tu Y, et al. (2019) Specific and Quantitative Detection of Albumin in Biological Fluids by Tetrazolate-Functionalized Water-Soluble AIEgens. ACS Appl Mater Interfaces 11(33):2961929629.

48. Zhang Y, Xie S, Zeng ZB, \& Tang BZ (2020) Functional Scaffolds from AIE Building Blocks. Matter 3(6):1862-1892.

49. Yang SL, Chou YT, Wu CN, \& Ho MS (2011) Annexin II binds to capsid protein VP1 of enterovirus 71 and enhances viral infectivity. $J$ Virol 85(22):11809-11820.

50. Pan M, et al. (2018) A reverse genetics system for enterovirus D68 using human RNA polymerase I. Virus Genes 54(4):484-492.

51. He TC, et al. (1998) A simplified system for generating recombinant adenoviruses. Proc Natl Acad Sci U S A 95(5):2509-2514.

52. Wei B, et al. (2015) Tom70 mediates Sendai virus-induced apoptosis on mitochondria. $J$ Virol 89(7):3804-3818.

\section{Acknowledgments}

This work was supported by grants from the National Key Research and Development Program of China (2017YFA0205102), Key Project of Tianjin Natural Science Foundation (19JCZDJC34100), National Natural Science Foundation of China (32000999 and 21788102), Research Grants Council of Hong Kong (16305518, C6009-17G and AHKUST 605/16), Innovation and Technology Commission (ITCCNERC14SC01), the Guangdong Provincial Key Laboratory of Luminescence from Molecular Aggregates (2019B03031003), Hunan Provincial Grant for Innovative Province Construction (2019RS2023), the Science and Technology Innovation Program of Hunan Province (2020RC5033), and the Natural Science Foundation of Hunan Province (2020JJ3003). The authors thank Xuanyuan Zheng of Tianjin Medical University for providing transmission electron microscopy (TEM) and the in vivo imaging system (IVIS). 


\section{Author contributions}

B.Z.T., S.X., and T.W. supervised the study. B.Z., X.L., and S.X. designed the study. S.X., S.W., W.L., Z.Z., Q.L., Y.Y., and Z.Z. designed and constructed the AIEgen library, and synthesized the PBET and other AIEgens for this study. B.Z., X.L., and B.H. performed the virus-related experiments related to developing, optimizing, and characterizing the fluorogenic labeling of viruses for cellular and animal imaging studies. W.N. and J.H. performed the molecular docking analysis. All authors contributed to the data interpretation and analysis. B.Z.T., S.X., T.W., B.Z., and X.L. wrote the manuscript with input from all authors.

\section{Data availability}

Supplementary materials to this article can be found online at https://. 